\title{
Understanding Higher Order Impacts of Green ICT
}

\author{
Albert Hankel \\ Department of Innovation and Environmental Studies \\ Utrecht University \\ Utrecht, The Netherlands \\ a.c.hankel@uu.nl
}

\begin{abstract}
ICT is known to have three orders of impact: direct, indirect and systemic. The first is often regarded as 'part of the problem', the latter two can also be 'part of the solution' to reduce the global environmental impact of human activities. A number of studies show that the impact of ICT itself is roughly $2 \%$, whereas smart use of ICT can reduce the impact of other sectors by up to $16 \%$. However, ICT research and practice seems to be mostly focused on the direct impacts. This paper tries to move away from this focus, explores the higher order impacts and presents a conceptual framework for individuals and organizations to make the higher order impacts more tangible.
\end{abstract}

Index Terms - Green ICT, Environmental impact of ICT, Greening by ICT, Framework

\section{Introduction}

As a general-purpose technology information and communication technologies can be used by themselves or as part of other technologies. This is the reason why ICT is viewed as an environmental friendly solution: even though the global environmental impact of ICT itself is significant (roughly 2\%), making something else more efficient has a much larger effect (potential reduction of $16 \%$ of the global footprint) [1]. This diverse set of impacts of ICT was recognized in 2001 by Berkhout \& Hertin [2], making a distinction between first order or direct effects, second order or indirect effects and third order or systemic effects. Others $[3,4]$ have defined these as:

- 'First order' or 'primary' effects: effects of the physical existence of ICT (environmental impacts of the production, use, recycling and disposal of ICT hardware).

- 'Second order' or 'secondary' effects: indirect environmental effects of ICT due to its power to change processes (such as production or transport processes), resulting in a modification (decrease or increase) of their environmental impacts.

- 'Third order' or 'tertiary' effects: environmental effects of the medium- or long-term adaptation of behavior (e.g. consumption patterns) or economic structures due to the stable availability of ICT and the services it provides.
Green ICT is the label that is used for all ICT actions that reduce the environmental impacts of human activities. Similar to the three orders of impact, there are three kinds of improvement: ICT itself can be made more efficient, ICT can be used to make other processes more efficient and finally it can be used to influence behavior by analyzing and sharing information. However, the Green ICT label is mostly used for the direct impacts only: it is usually focused on efforts that make ICT more energy efficient or reduce the environmental impact of ICT itself in other ways. This is illustrated by a definition of Green ICT that is often quoted [5]:"It's the study and practice of designing, manufacturing, using and disposing of computers, servers, and associated subsystems ... efficiently and effectively with minimal or no impact on the environment". Furthermore, in practice most efforts in 'Green ICT' go towards actions related to the direct impact, such as greening the datacenter and power management of ICT devices.

Whenever technology is used as a solution for an environmental problem, ICT is often part of it. For example, to enable next generation power grids - the smart grid (the name says it all) - ICT is a necessary component to realize efficient local, regional and national trading of energy. But it seems, from an ICT perspective, a field of its own and mostly driven by energy experts. A risk might be that ICT is implemented as a necessity rather than an optimization factor; or, in positive terms, the ICT world is missing an opportunity tot promote itself as an environmental friendly solution. Another example: it often happens that whenever new buildings are built, these are not optimized for the role ICT could have (e.g. domotica), because ICT experts are not involved in the design, but only after the building is completed.

Why is it that whenever ICT researchers and professionals discuss the environmental impact of ICT, most of the efforts go towards the first order impacts? If the effects of ICT as a solution can be that much greater than reducing the problematic side of ICT, it makes sense to shift efforts from reducing the $2 \%$ to help reducing the other $98 \%$. From practical experience, it seems that the main reason for this is that it is easier to focus on the direct impacts: it is quantifiable, relationships between change and effect are much shorter and things are therefore less complex. Another important factor is responsibility: the energy sector is responsible for reducing the impact of the energy sector, the construction sector is responsible for reducing the impact of 
buildings and the ICT sector is responsible for reducing the impact of ICT. At the organizational level, similar things happen between departments. There is little incentive to look outside the department walls (because of split budgets for example), but it is clear that a fully optimized ICT department or a Facility department is not the same as a fully optimized organization in terms of environmental impact.

The question is how to make the ICT departments see the wider picture, how to make them feel responsible and how to get them to act. In short: how can we make the higher order impacts more tangible for ICT experts (in organizations)? In this paper I will explore this question by analyzing categories of effects of these higher order impacts. This analysis is then translated to a framework that should help individuals and organizations, but also future work, to make the second and third order impacts more tangible.

\section{An overview of effects}

The starting point for analyzing the higher order impacts of ICT is a conceptual framework presented by Hilty [6]. Ignoring the first order effects, there are six effects mentioned, being: optimization effects, substitution effects, induction effects, deep structural change toward a dematerialized economy, rebound effects and new critical infrastructure. In addition to these there are a number of other effects mentioned in literature, such as in the SMARTer2020 report [1]. This report defines four change levers: digitalization and dematerialization, data collection and communication, system integration, and process, activity and functional optimization. Further effects attributed to higher impacts of ICT are: obsolescence effects, rematerialization effects and network effects $[7,8]$.

I think these effects capture the most important aspects of the higher order impacts of ICT. Do note that there is some overlap between them, as we shall see below. What is distinct about the second and third order impacts from the first order impacts is that they usually require a behavior change or have some impact on behavior. This interaction between technology and behavior, the socio-technological aspect, is I think key to a better understanding. Let's take a closer look at them.

\section{A. Optimization effects}

Optimization effects may occur "in all phases of the life cycle, as well as in the design phase. CAD tools, for example, can be used to optimize a product for environmental criteria (eco-design). Design has a strong impact of the life cycle

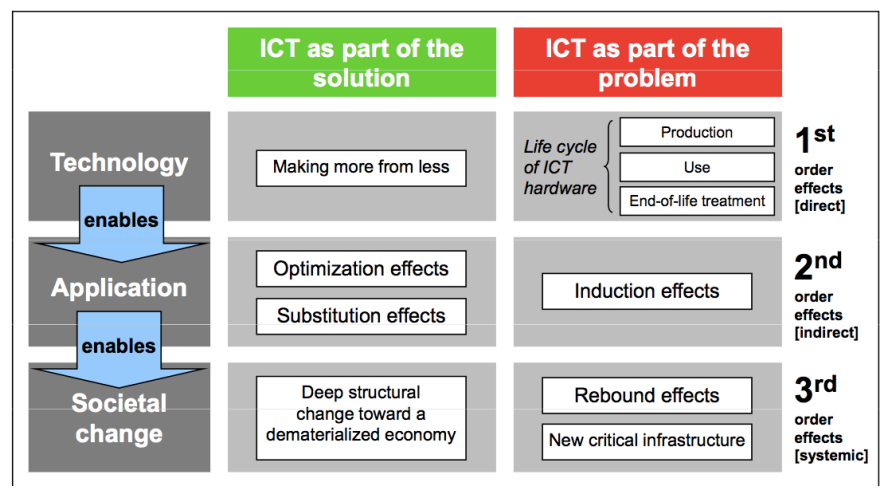

Figure 1 Conceptual Framework of ICT impacts (Hilty, 2008). because it constrains the optimization potentials that will exist in the production, use and end-of-life phases. For example, if the variety of materials or the complexity of the product can be reduced in the design phase, it will be possible to reach a higher efficiency level in end-of-life treatment" [7]. Here ICT can have a positive effect because it can make processes, products and services more effective by automating steps (let ICT make decisions) or making conversions (e.g. digital material - digital) disappear. Broadly speaking, optimization can have effect on time (the same process can be done faster), costs (the same process can be done cheaper) and environmental impact (the same process has less environmental impact). Of course optimization changes often do not lead to singular effects but are rather a combination of these.

\section{B. Substitution effects}

Substitution effects occur "when an ICT service replaces the use of a physical product, e.g. when e-mail replaces the use of conventional paper-based mail" [7]. This positive effect is profound when the ICT service is functional equal or superior. Dematerializing goods such as books and music are the first that come to mind. Often these substitutions are functionally not entirely the same, which may lead to incomplete substitutions. Take for example videoconferencing, which is not exactly the same as face-to-face meetings. Organizations might install videoconferencing equipment and still have people traveling almost as much as they did before [9]. Substitution effects are a question of adoption: how do users respond to a functional change within the process? They might not want to use the ICT substitute because it is functionally weaker in some aspects (incomplete substitution), they might be indifferent or simply accept the change (complete substitution) and they might use the ICT substitute more because of new additional features (overcomplete substitution). These usage patterns have different effects on the environment.

\section{Induction effects}

Induction effects occur "when an ICT service stimulates the use of the other product, i.e. more functional units per unit of time are consumed (e.g. the text-processing service provided by a PC system with a printer may stimulate paper consumption)" [7]. Contrary to rebound effects, the induction effect is purely based on a response to some functional change that may lead to extra use of other products.

\section{Deep structural change toward a dematerialized economy}

Traditionally, an economy was usually represented as the value of natural resources, labor and capital. Growth depended on the ability to utilize more resources or labor, but more recently this simple description was found to be lacking an essential component, namely skills, knowledge and information. With the rise of ICT use, another 'immaterial' component became visible - that of digital or virtual goods. While there is a certain dependency on material resources, which have a direct link to environmental impact, the move towards immaterial goods does not necessarily lead to more impact. 
The assumption here is that ICT is an enabler for decoupling economic growth from growth in use of natural resources (material and energy), a.k.a. environmental impact. It is possible that ICT stimulates a move towards an economy where we could in this way (globally) limit the use of natural resources. Extra value for the economy is created only through the creation of immaterial goods.

It seems that such a structural change will not happen by itself and needs to be imposed on 'from above' through policies and regulation. Of course ICT stimulates the use of immaterial goods, but often the freed up material resources are then used in other ways. In other words: it is hard to imagine that this change happens automatically because users and organizations are motivated and incentivized to limit themselves only to value creation through digital goods, at least as long as there are free common resources such as air (tragedy of the commons).

\section{E. Rebound effects}

Rebound effects in general are all effects that are a (system) response to introduced change by ICT. In its simplest form it is a behavior change that followed a certain introduction of technology, for example an optimization or a substitution. Note that rebound effects can be both positive and negative. However, the rebound effect has become synonymous with a phenomenon known as the Jevons' Paradox. Already in 1865, Jevons found a pattern in coal consumption that an increase in efficiency leads to an increase in consumption. Generally speaking, there seems to be a relationship between efficiency and consumption. Simply put: an increase in efficiency leads to lower costs which leads to lower prices which leads to increased demand. This increase in demand can be caused by current users consuming more than before or new users who can now afford consumption.

Similar patterns are imaginable when ICT is introduced in a process or as a new technology. This effect may be especially profound when something becomes so efficient it is almost free (think e-mail versus regular mail). If something becomes more efficient, it generally saves time or money and it makes sense to ask the question what happens with that time or money on a system level [10]: do we use it to send more e-mails or do we invest it in other activities? How environmentally friendly are these activities?

A more complex form of the rebound effect is when something becomes so efficient it enables new forms of technologies, behavior or processes - innovation basically. There are thresholds or tipping points that mark this enabling point and this change could lead to either a positive or a negative environmental impact. These innovations can either be known or unexpected.

\section{F. New critical infrastructure}

As with all technologies that become an essential part of our lives, ICT introduces an infrastructure that we depend upon in our daily lives. For most of us, it is hard to imagine a life without the Internet. In addition, since ICT is a general-purpose technology, it becomes part of other infrastructures as well, such as energy and transport infrastructures. The ICT infrastructure and its interconnectedness with our daily lives lead to two kinds of criticalities: how vulnerable it is and how easily it can be replaced.

Hilty [7] links vulnerability to complexity and whether it is mastered or not. Specifically, unmastered complexity, translated to not fully understanding software code and its interaction with the underlying (networked) hardware, leads to vulnerabilities because it is impossible to assess if the risk on failure and abuse is acceptable.

The second criticality is more linked to innovation and future change. Whereas a new infrastructure is an enabler for all kinds of innovations, legacy infrastructure can be an obstruction. Take for example the power grid, which is designed for few and large production facilities that provide power to consumers (unidirectional) and is not designed for large numbers of consumers that also want tot produce small amounts of energy and deliver any excesses back to the network (bidirectional). This property of the power infrastructure is obstructing a fast change towards a smart grid. This was not foreseen when the old infrastructure was designed and something similar could happen to the ICT infrastructure.

\section{G. Digitalization and dematerialization}

The SMARTer2020 report takes a different angle with the next four change levers (G-J). Their description for digitalization and dematerialization is "substituting or eliminating the need for an emissions-intensive product, material process, or service. Also the reuse/multiple use of information sources, media, etc. via ICT" [1]. Examples here are videoconferencing, telecommuting, online media and ecommerce.

This change lever seems more or less identical to the substitution effect mentioned above with a focus on a one-toone comparison in terms of impact on the environment. Success of this change lever largely depends on the completeness of the substitution. There are some other effects on a system level that should be taken into account however. Take for example e-commerce where the need for stores and showrooms are eliminated, but extra transportation is required.

\section{H. Data collection and communication}

Data collection and communication is described as "providing real-time data and analysis that allows for better decision making, identifies a need for change, or encourages more efficient behaviors" [1]. Examples here are time-of-day pricing (of energy), real-time traffic alerts, asset sharing and using weather forecasts for soil management.

The availability of the right information at the right time, the granularity of information and the ability to process large amounts of information quickly are all enablers (provided by ICT) for these examples. Basically, the assumption is that the better knowledge we have about the system state and impact of our choices, the more able we are to make efficient decisions that in turn should relieve pressure on the environment.

\section{System integration}

System integration is described as "managing the use of resources and integrating lower-emissions intensive processes" 
[1]. Examples given are integration of renewables, virtual power plant, fleet management \& telematics, building management system.

This change lever seems to be a combination of optimization and system knowledge through data collection and analysis. There is a strong pattern in the examples here to centralize resources and then distribute them again in the most efficient manner. The difference with the previous change lever 'data collection and communication' is that this system optimization is done automatically within parameters set by users.

\section{J. Process, activity and functional optimization}

Finally, the description for the last change lever 'Process, activity and functional optimization' is 'intelligent simulation, automation, redesign, or control to optimize process, activity, function, or service" [1]. Examples here are power-load balancing, optimization of truck route planning and minimization of packaging.

This change lever seems rather identical to the optimization effect described above.

\section{K. Obsolescence effects}

ICT products and services can lead to the planned or unplanned rapid obsolescence of other products, within ICT (think mobile phones \& subscriptions or hardware-software interactions) or outside the ICT realm through substitution effects for example. The obsolescence effect is in some ways opposite to induction in the sense that a functional change leads to decreased use of other products.

In addition, ICT components in products may transfer their rapid lifecycle to the whole product. When ICT becomes part of common household products for example, it may introduce new functionalities as well as extra complexity. Both could cause a speed up in the replacement cycle, e.g.: demand for new functionalities that are not introduced on the old model; or increase in maintenance costs which rebalance the choice between repair and replace.

Finally, if an ICT change results in time and cost efficiency, these benefits may also lead to obsolescence effects, mainly because it may become cheaper to replace a product rather than repair it (especially when extra functionalities are taken into account).

\section{Rematerialization effects}

Rematerialization is in a sense the opposite of dematerialization: virtual goods become physical goods (again). Printing is of course the most obvious example where electronic texts are made into texts printed on paper. Sometimes printing is planned and the process is actually having less impact than before because the input (e.g. writing a manuscript) has been dematerialized, but not the output (book). Often rematerialization is not planned by the one delivering the input, take printing e-mails for example. Most senders of emails do not expect their e-mails being printed by the receivers, yet this is common practice in some areas.

With 3D-printing the scope of goods that can be (re)materialized becomes much broader. Ideally, 3D-printing substitutes for process steps (and saves transportation costs) in the manufacturing of goods we would have bought anyway. But it is imaginable that we will print beyond that; that digital goods that were never meant to be physical goods will be printed.

Rematerialization is not caused by efficiency gains or saving effects, but rather the opposite. Having the physical good instead of the digital one has some added benefit that we would like to 'pay' for it and cause additional environmental impact. Rematerialization can be seen as a form of induction.

\section{Network effects}

One of the fundamental impacts of the ICT infrastructure on our daily lives is that it increases connectivity in the broadest sense. Everything from the physical to the social is becoming more connected. This opens up the world, literally.

Most of the effects described here, relate to input and output (time, costs, resources, environmental impact) and how they move around the system. Increased connectivity affects these movements in terms of scope and granularity: it affects choice.

Examples of this increase in choice are plentiful: thanks to the Internet, we can do price comparisons more easily and are less bound to regional choices. With online auctioning and second use marketplaces, it becomes easier to find both new users for our old stuff as well as find components to fix something broken. ICT allows us to create tools to share information on use more easily, which in turn enables us to share goods we do not use all the time, like car sharing. On the other hand, social networks become more global as well, giving us incentives to do business beyond our borders and visit places we may not have had otherwise (friends abroad, tourism) [8].

In short, network effects may increase the lifetime and utilization of goods and it may let us make more informed but also more global decisions. The network effects can have both positive and negative impact on the environment.

\section{Towards a conceptual framework}

The effects mentioned in the previous section may not be all effects ICT might have, but they do capture the most mentioned and probably the most important ones. There is some conceptual overlap between effects, but together they provide a good starting point for further analysis. In the end it boils down to people that want to do something and that action has some impact on the environment (many complex system responses can emerge after such an action or a multitude thereof; the action is a good starting point for a framework, though). I am looking for the role that ICT plays here, as a good itself but also how it affects these actions and the interplay between people and actions over the course of time.

In general it seems that ICT can have an effect on the quality, cost, material resources and time of an action. In addition ICT can influence the decisions people make by collecting and analyzing information. Furthermore these effects are not limited to the action or good itself, but also apply to substituted or replaced goods, other goods in the context or action space, how long a good is used and the system in its 
entirety. Other patterns are more centered on the user with main questions like what happens with the extra time and money one has if ICT has made something more efficient.

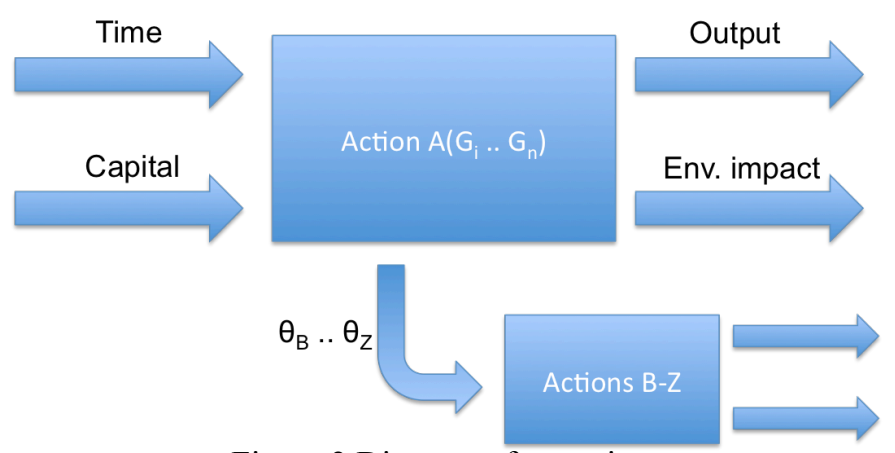

Figure 2 Diagram of an action.
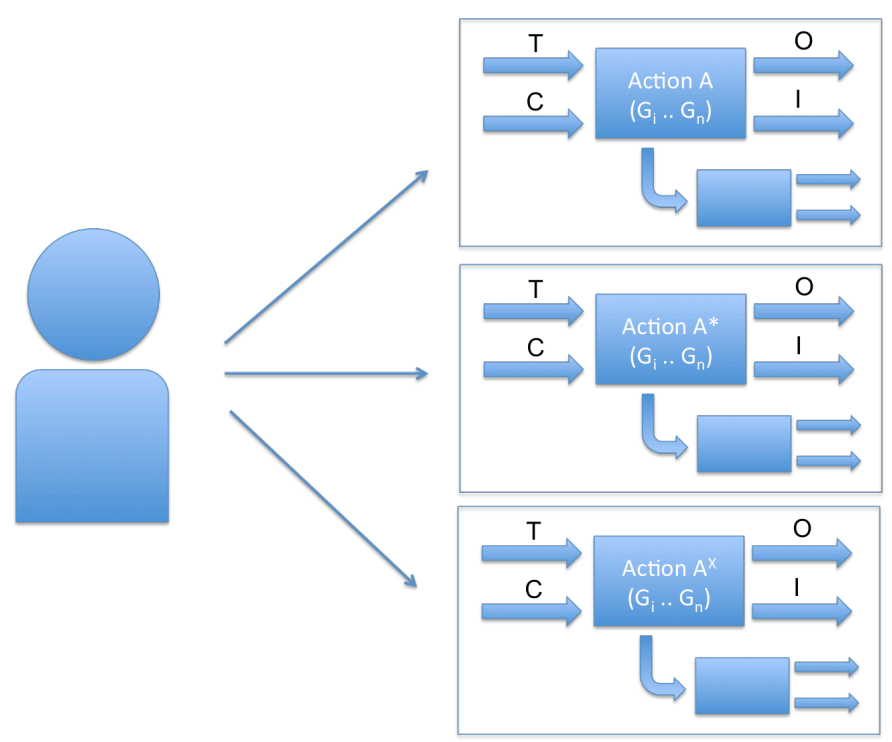

Figure 3 An actor's choice between actions.

Translating all these aspects to a conceptual framework starts with an action that an individual or an organization (henceforth: actor) carries out to achieve a certain output. The general form is described in figure 2. To carry out action A which results in a desired output $\mathrm{O}$ and has an environmental impact $I, n$ goods are required $\left(G_{i} . . G_{n}\right)$. An actor needs to invest some time $\mathrm{T}$ and capital $\mathrm{C}$ to be able to carry out that action $\mathrm{A}$. Whenever $\mathrm{A}$ is carried out, it stimulates other actions $\mathrm{B}-\mathrm{Z}$ with a certain threshold $\theta$. This is the baseline to which we can compare the effects of a change introduced by ICT. Note that the output of an action is quite complex: it has many different properties that can all be changed. The assumption here is that when choosing between two actions to achieve a desired output, the difference between the two O's is simple enough to value it and make a decision.

The goal of Green ICT is to change the action A in such a way that the environmental impact I is reduced. Here this new action is described as $\mathrm{A}^{*}$. An actor can choose between $\mathrm{A}$ and $A^{*}$ (and $A^{x}$, which is explained later on) as shown in figure 3 . To determine whether action $\mathrm{A}^{*}$ is preferred above action $\mathrm{A}$ depends on the differences in inputs and outputs. Expressed in a formula, it would look like this:

$$
\mathrm{w}_{\mathrm{T}}\left(\mathrm{A}_{\mathrm{T}}-\mathrm{A}_{\mathrm{T}}^{*}\right)+\mathrm{w}_{\mathrm{C}}\left(\mathrm{A}_{\mathrm{C}}-\mathrm{A}_{\mathrm{C}}^{*}\right)+\mathrm{w}_{\mathrm{O}}\left(\mathrm{A}_{\mathrm{O}}^{*}-\mathrm{A}_{\mathrm{O}}\right)+\mathrm{w}_{\mathrm{I}}\left(\mathrm{A}_{\mathrm{I}}-\mathrm{A}_{\mathrm{I}}^{*}\right)>0
$$

Each actor can weigh all factors (time, cost, output and environmental impact) with weights $\mathrm{w}$ according to their own preferences. Note that the alternative factors for time, cost and impact should be lower as one would want to reduce those, while the reverse is true for the output where one would want better quality. The concept behind this framework is that ICT can affect all these factors. In fact, it does not matter whether the change was intentionally green or not.

In addition, ICT can inform an actor about his or her choices. Because most of the environmental impact is currently not paid for, these are externalized. What this means is that the environmental impact is not taken into account when making a decision (i.e. $\mathrm{W}_{\mathrm{I}}=0$ or $\mathrm{A}_{\mathrm{I}}-\mathrm{A}_{\mathrm{I}}^{*}=$ unknown). ICT can inform actors about the environmental impact of possible actions that lead to the desired output $\mathrm{O}$ and in this way change the outcome of the formula (the environmental impact is now actually calculated and weighted instead of not used at all) and thus influence decision making.

Finally, ICT can also inform an actor of an alternative course of action $\mathrm{A}^{\mathrm{x}}$ with different goods that may lead to similar output $\mathrm{O}$ but with less environmental impact (not necessarily because ICT was used to lower the impact).

The environmental impact of an action depends mostly on the goods used to carry out the action and the way they are used. This can be expressed as the sum of the environmental impacts of the goods:

$$
\mathrm{A}_{\mathrm{I}}=\Sigma_{\mathrm{i} . \mathrm{n}} \mathrm{I}_{\mathrm{Gi}}
$$

The environmental impact of a good $\mathrm{G}$ used in action $\mathrm{A}$ can be derived from their life cycle, with stages design $\mathrm{D}$, production $\mathrm{P}$, use $\mathrm{U}$, and end-of-life $\mathrm{E}$ :

$$
\mathrm{I}_{\mathrm{G}}=1 / \mathrm{n} * \mathrm{D}+1 / \mathrm{n} * \mathrm{P}+\mathrm{U}_{\mathrm{A}}+1 / \mathrm{n} * \mathrm{E}
$$

In this formula $\mathrm{n}$ is the number of times a good can be used before it needs to be replaced and $\mathrm{U}_{\mathrm{A}}$ is the specific impact of using good $\mathrm{G}$ in action A (e.g. energy consumption).

\section{An explanation of effects with the framework}

The framework presented in figures 2 and 3 should be able to explain most of the effects mentioned in section II. I will review them one by one.

In optimization effects, optimizing an action more or less means doing something more efficient and in the context of this framework it means keeping the output the same while reducing time, cost or environmental impact. Goods are not changed but for example the usage is made more efficient or their longevity is extended. In formula 1 this means that $\mathrm{A}^{*}{ }_{\mathrm{O}}$ $A_{O}$ equals to zero, but (a combination of) the other factors, for example $A_{T}-A^{*}$, should be greater than zero to make an actor choose $A^{*}$. Note that if time or costs are reduced in $A^{*}$ but the 
environmental impact stays the same, the systemic effect is an increase of impact because on average the extra time and capital will be reinvested in some non-zero impact activity. So for an optimization effect to be environmental friendly (and to avoid some rebound effects), the following should hold, where $I_{a v g}$ is the average impact per unit of time or capital in general:

$$
A_{I}-A_{I}^{*}>I_{a v g}\left(A_{T}^{*}-A_{T}\right)+I_{a v g}\left(A_{C}^{*}-A_{C}\right)
$$

Substitution effects are more targeted towards changing goods $\mathrm{G}$ used in an action. Non-ICT goods are replaced by ICT goods that may or may not deliver the same output $O$. Whether an actor accepts this alternative action $\mathrm{A}^{*}$ depends on the balance between change in quality and functionality and the achieved reduction in time, cost and environmental impact:

$$
\mathrm{w}_{\mathrm{T}}\left(\mathrm{A}_{\mathrm{T}}-\mathrm{A}_{\mathrm{T}}^{*}\right)+\mathrm{w}_{\mathrm{C}}\left(\mathrm{A}_{\mathrm{C}}-\mathrm{A}_{\mathrm{C}}^{*}\right)+\mathrm{w}_{\mathrm{I}}\left(\mathrm{A}_{\mathrm{I}}-\mathrm{A}_{\mathrm{I}}^{*}\right)>\mathrm{w}_{\mathrm{O}}\left(\mathrm{A}_{\mathrm{O}}-\mathrm{A}_{\mathrm{O}}^{*}\right)(5)
$$

Induction and rematerialization effects in this framework equal an increase in other actions (B-Z) caused by a functional change in action $A^{*}$. In other words, the difference between $\mathrm{A}_{\mathrm{O}}{ }_{\mathrm{O}}-\mathrm{A}_{\mathrm{O}}$ had some impact that lowered the thresholds $\theta_{\mathrm{B}} . . \theta_{\mathrm{Z}}$ to a point that these other actions are carried out more often compared to using action A.

The change lever data collection and communication refers to making the 'right' choice. If an actor is informed of the time and cost required and the environmental impact of all possible actions that achieve a satisfactory output $\mathrm{O}$, the actor can the make a decision (likely that action that has the highest result from formula 1).

System integration is somewhat hidden in the framework but could be interpreted as a combination of optimization of goods and adding extra ICT goods. For this to be a viable alternative to an actor, formula 4 should hold. This effectively means that the extra good $\mathrm{G}_{\mathrm{ICT}}$ changes the impacts (on time, costs and environment) of the other goods in such a way that the net result contributes to a better result for formula 4 .

Obsolescence effects of action $A^{*}$ are almost always present in conjunction with other (positive) effects because otherwise an actor would have no reason to choose $\mathrm{A}^{*}$ over A. They occur either when a good has been replaced to be able to do A* or when the extra complexity introduced by ICT lowers the number of times a good can be used (i.e. it lowers $n$ in formula 3).

Network effects seem quite similar to 'data collection and communication' but are in fact much broader. Because it adds more choice, it can affect goods in several ways: it can lower the environmental impact of the end-of-life phase or it can increase the number of times $n$ a good is used (formula 3 ). In addition, being able to share goods with others can lower costs.

\section{Discussion and future work}

The framework presented here is able to explain most of the environmental effects ICT may have found in literature. Because ICT is such a general-purpose technology, it can impact an action at almost any point in the process. As the effects described in section II show, they are often the result of a combination of factors time, cost, output and environmental impact.

The goal was to make the effects more tangible: we know they are there, but what makes them tick? It is insightful to deconstruct them into these factors, because it then becomes possible to derive formulas that express the balance between these factors, such as formula 4 and 5 . These formulas can then empower ICT departments and organizations to become a proactive partner in reducing the environmental impact using Green ICT.

From a research point of view, the framework can also be used to see if there are any combinations of factors that result into new effects that are currently not visible (in practice and in research).

Of course the current framework is not quite ready yet for this supportive role. First of all, it does not capture all effects but is mostly focused on second order impacts. The system and therefore systemic impacts are for a large part not yet included. Second, the framework needs to be validated by empirical evidence. The framework is currently based on logics derived from literature and personal experiences. This was enough to create the framework but not to validate it. Relationships between concepts should be tested as well as the formulas. It may also be possible that there are extra factors that should be taken into account.

Future work will include both the expansion of the framework to include systemic impacts as well as validation of the framework. Because it should help decision-making, ease of use and the ability to quantify factors should also be tested (note that it is often sufficient to be able to quantify the difference between action $\mathrm{A}$ and $\mathrm{A}^{*}$ ). Lessons for the latter can be derived from life cycle analysis literature, where the dynamics of input and output factors have been studied in depth. In addition the relationships and factors in the framework will be investigated more deeply by doing a number of case studies on ICT projects and goods. Finally, the usefulness of the framework will be tested by applying it to a significant number of (Green) ICT vs. non-ICT actions from practice.

\section{Conclusion}

In this paper I have presented an overview of possible higher order impacts that ICT could have on the environment. Some key characteristics and patterns were derived from this overview to create a conceptual framework. These key characteristics relate to general concepts like time, costs, output, environmental impact and information availability. The framework is able to explain several higher order environmental impacts of (green) ICT. Because this explanation is offered from the point of view of an actor, it makes these impacts more tangible for ICT practitioners who could use such a framework to promote a viable Green ICT alternative. Before that can happen, the framework should be further explored and validated. 


\section{References}

[1] GeSI (2012). SMARTer2020: The Role of ICT in Driving a Sustainable Future. http://gesi.org/SMARTer2020

[2] Berkhout, F., \& Hertin, J. (2001). Impacts of information and communication technologies on environmental sustainability: Speculations and evidence. Report to the OECD, Brighton, 21.

[3] Köhler, A., Erdmann, L. (2004). Expected environmental impacts of pervasive computing. Human and ecological Risk Assessment, 10(5): 831-852.

[4] Hilty, L.M., Arnfalk, P., Erdmann, L., Goodman, J., Lehmann, M. and Wäger, P.A. (2006a). The relevance of information and communication technologies for environmental sustainability A prospective simulation study. Environmental Modelling \& Software, 21(11): 1618-1629.

[5] Murugesan, S. (2008). Harnessing Green IT: Principles and Practices. IT Pro, (Jan/Feb): 24-33.

[6] Hilty, L. M. (2008, May). Environmental impact of ICT-A conceptual framework and some strategic recommendations.
OECD workshop on ICT and environmental challenges Copenhagen.

[7] Hilty, L. M. (2010). Information and Communication Technologies for a more Sustainable World. Green Technologies: Concepts, Methodologies, Tools and Applications, 36.

[8] Linderhof, V., van der Bergh, J., \& Verbruggen, H. (2006). Digital Dematerialisation: Economic Mechanisms behind the Net Impact of ICT on Materials Use. M. Salih, J. Cramer \& L. Box (Ed. 's), Climate Change and Sustainable Development New Challenges for Poverty Reduction. Cheltenham: Edward Elgar Publishing.

[9] Røpke, I. (2012). The unsustainable directionality of innovation-The example of the broadband transition. Research Policy, 41(9), 1631-1642.

[10] Erdmann, L., Hilty, L., Goodman, J., \& Arnfalk, P. (2004). The future impact of ICTs on environmental sustainability. Institute for Prospective Technological Studies. 